Article

\title{
Influence of Swing-Foot Strike Pattern on Balance Control Mechanisms during Gait Initiation over an Obstacle to Be Cleared
}

\author{
Romain Artico $^{1,2,3}$, Paul Fourcade ${ }^{1,2}$, Claudine Teyssèdre ${ }^{1,2}$, Teddy Caderby ${ }^{4}$, \\ Arnaud Delafontaine ${ }^{1,2}$ and Eric Yiou 1,2,* \\ 1 CIAMS, Université Paris-Saclay, 91404 Orsay, France; romain.artico@u-psud.fr (R.A.); \\ paul.fourcade@u-psud.fr (P.F.); claudine.Teyssedre@u-psud.fr (C.T.); arnaud.delafontaine@u-psud.fr (A.D.) \\ 2 CIAMS, Université d'Orléans, 45067 Orléans, France \\ 3 Ecole Nationale de Kinésithérapie et Rééducation, 75012 Saint Maurice, France \\ 4 IRISSE Laboratory, Université de la Réunion, 97430 Le Tampon, Ile de la Réunion, France; \\ teddy.caderby@univ-reunion.fr \\ * Correspondence: eric.yiou@u-psud.fr; Tel.: +33-169-153-159; Fax: +33-169-156-222
}

Received: 29 October 2019; Accepted: 20 December 2019; Published: 28 December 2019

\begin{abstract}
Gait initiation (GI) over an obstacle to be cleared is a functional task that is highly challenging for the balance control system. Two swing-foot strike patterns were identified during this task-the rearfoot strike (RFS), where the heel strikes the ground first, and the forefoot strike (FFS), where the toe strikes the ground first. This study investigated the effect of the swing-foot strike pattern on the postural organisation of GI over an obstacle to be cleared. Participants performed a series of obstacle clearance tasks with the instruction to strike the ground with either an FFS or RFS pattern. Results showed that anticipatory postural adjustments in the frontal plane were smaller in FFS than in RFS, while stability was increased in FFS. The vertical braking of the centre of mass (COM) during GI swing phase was attenuated in FFS compared to RFS, leading to greater downward centre of mass velocity at foot contact in FFS. In addition, the collision forces acting on the foot were smaller in FFS than in RFS, as were the slope of these forces and the slope of the C7 vertebra acceleration at foot contact. Overall, these results suggest an interdependent relationship between balance control mechanisms and foot strike pattern for optimal stability control.
\end{abstract}

Keywords: anticipatory postural adjustments; balance control; foot strike pattern; gait initiation; obstacle clearance; biomechanics

\section{Introduction}

Like all terrestrial species, humans move in a gravity field that permanently attracts them towards the centre of the earth. Therefore, balance control is a key component of daily motor tasks. Gait initiation (GI), which corresponds to the transient phase between quiet standing and level walking, is a classical paradigm for investigating how balance is controlled during a functional whole-body movement [1,2]. Gait initiation can be broken down into a postural phase (corresponding to anticipatory postural adjustments (APAs)), followed by a swing phase that ends at the time of swing foot contact with the support surface. These APAs are characterised by a centre of pressure (COP) shift backwards towards the heels, and laterally towards the forthcoming swing leg. The backward centre of pressure shift reflects a strategy to generate the initial propulsive forces necessary to reach the intended centre of mass velocity at the end of gait initiation [3-6]. The mediolateral (ML) centre of pressure shift reflects a strategy to propel the centre of mass above (or beneath) the forthcoming stance foot in order to maintain ML stability during the swing phase of gait initiation [7-11]. 
Besides ML APAs, a major mechanism involved in stability control is the braking of the centre of mass fall during the swing phase of gait initiation. Previous lines of research have shown that braking is an active phenomenon that occurs in anticipation of the swing foot's collision with the ground, and involves the activation of the soleus of the stance leg [12-15]. Anticipatory centre of mass braking attenuates the vertical ground reaction forces (GRFs) at the time of foot collision (hereafter called collision forces), thus facilitating balance control and reducing the risk of injury linked to the transmission of this impact throughout the musculoskeletal system.

These collision forces are also known to depend on the swing-foot strike pattern at the time of collision [16-18]. At least two main swing-foot strike patterns have been identified in the literature-rearfoot strike (RFS), where the swing heel strikes the ground first, and forefoot strike (FFS), where the swing toe strikes the ground first [16]. Because studies have reported that participants systematically used an RFS pattern, foot strike patterns have so far not been considered in the literature on gait initiation. However, a recent study showed that when gait was initiated with the goal of clearing an obstacle longer than $30 \%$ of body height, a change from an RFS to an FFS pattern was observed in most trials [10]. Based on previous research on the effect of the foot strike pattern on collision forces during stepping down while walking [17,18] or running [16,19-22], it was proposed that this change in foot strike pattern reflected a strategy directed at attenuating collision forces.

It is surprising that the authors of these important lines of research, focusing on foot strike pattern effects, did not consider that the magnitude of the anticipatory braking might have changed between the RFS and FFS patterns. Indeed, such a change could at least partly account for the differences in the collision forces observed, with a larger anticipatory centre of mass brake being potentially responsible for greater attenuation of the collision forces. More generally, a brief literature review shows that the balance control mechanisms described above have been investigated by independent lines of research (for a recent review see [2]). Thus, it remains largely unknown whether these mechanisms (i.e., ML APAs, force braking and foot strike pattern) are independently controlled by the central nervous system (CNS) or are interdependent. Gait initiation with an obstacle to be cleared is a natural task that offers the opportunity to test the interdependence between these mechanisms since, as stated above, both foot strategies are naturally used to land on the support surface.

In the present article, it is expected that the ML APAs and the vertical centre of mass braking mechanisms are programmed according to foot strike patterns (FFS or RFS). Specifically, it is reasoned that the need to actively brake the centre of mass fall during the step-swing phase becomes less imperative in FFS if the FFS pattern is more effective than the RFS pattern to attenuate the collision forces, as repeatedly shown in the literature [16,19-22]. Because it has an energetic and attentional cost [12], the CNS may consequently choose to attenuate this active braking for economical purposes. If so, the duration of the swing phase of gait initiation can be expected to be shortened for the FFS pattern, since the centre of mass would fall more rapidly.

Of particular interest, recent studies reported that the amplitude of the ML APAs associated with gait initiation over an obstacle was programmed according to this temporal parameter (i.e., the duration of the swing phase), and was lower when this duration decreased with a lower obstacle $[10,11]$. In the present study, changes in ML APAs amplitude can therefore be expected because of the changes in the swing-foot strike pattern.

Hence, the present study aimed to investigate the effect of changing the swing-foot strike pattern (FFS vs. RFS) on the postural organisation of gait initiation over an obstacle. The hypothesis of an interdependent relationship between balance control mechanisms for optimal stability control was raised. Specifically, and for the reasons detailed above, it was expected that both active braking of the centre of mass fall and the ML APAs associated with gait initiation over an obstacle to be cleared would be attenuated in the FFS compared to the RFS pattern, but with no consequent alteration in ML stability or increase in collision forces. 


\section{Materials and Methods}

\subsection{Participants}

In all, 13 young healthy subjects (eight males and five females, age (mean standard \pm 1 deviation), $28.7 \pm 1.5$ years; height, $178.1 \pm 7.2 \mathrm{~cm}$; weight, $69 \pm 8.3 \mathrm{~kg}$ ) participated in the experiment. All were free of any known neuromuscular disorders. They provided written informed consent after being informed of the nature and purpose of the experiments, which were approved by the local ethics committee of University Paris-Sud, Paris-Saclay (EA 4532, 10 September 2019). The study complied with the standards set by the Declaration of Helsinki.

\subsection{Experimental Protocol}

The participants were instructed to initiate gait at maximal velocity with their preferred limb while clearing an obstacle, and to continue walking at the same velocity to the end of a five-meter track (Figure 1). The task was self-triggered, that is, the participants initiated gait only when they felt ready. As in our previous study [11], the obstacle height was $10 \%$ of the subject's height and its distance from the subject was 30\% of the subject's height. Two conditions of gait initiation were performed. Under the first condition, the participants were instructed to strike the ground with the heel first after stepping over the obstacle (rearfoot strike (RFS) condition). Under the second condition, they were instructed to strike the ground with the forefoot first (forefoot strike (FFS) condition). We showed in our previous study [11] that participants used both the FFS and the RFS patterns under the condition where the obstacle had the same distance/height features as in the present study. In other words, no clear preferred foot strike pattern could be detected, suggesting that participants of the present experiment were not much constrained by the instruction on the foot strike pattern.

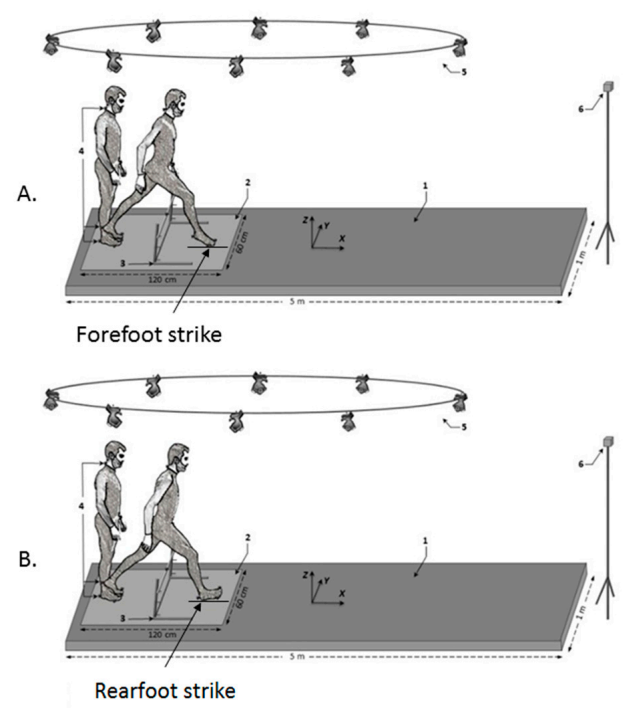

Figure 1. Schematic illustration of the experimental setup. (A) Gait initiation with a forefoot strike (FFS) pattern. (B) Gait initiation with a rearfoot strike (RFS) pattern. 1, walkway; 2, force plate; 3, obstacle; 4, reflective marker; 5, Vicon camera; 6, visual target.

The order of the conditions was randomised to avoid rank effects. For each condition, the subjects were allowed two familiarisation trials, after which five trials were recorded. In the initial posture, the participants stood upright barefoot, with their feet hip-width apart, their arms hanging loosely alongside their body and their body weight evenly distributed between their legs.

Gait was initiated on a force plate $(600 \times 1200 \mathrm{~mm}$, AMTI, Watertown, MA, USA) positioned at the beginning of the track. The force plate was embedded in the track and was long enough so that the participant's swing foot always landed on it at the end of gait initiation. The obstacle consisted of 
a lightweight wooden rod (length, $65 \mathrm{~cm}$; diameter, $1 \mathrm{~cm}$ ) resting on two adjustable upright stands (Figure 1). The participant's toes served as the reference point for positioning the obstacle. Reflective skin markers ( $9 \mathrm{~mm}$ in diameter) were placed bilaterally at the hallux (toe markers), head of the fifth metatarsal (metatarsal markers) and posterior calcaneus (heel markers). In 10 subjects, a marker was also placed at the top spine level (C7) to investigate the transmission of the collision forces from the swing foot to the upper part of the musculoskeletal system. An additional marker was positioned at the middle of the obstacle to determine the safety distance, that is, the distance between the swing foot and the obstacle at the time of clearance. A V8i VICON eight-camera (Mcam2) motion capture system (Oxford Metrics Ltd., Oxford, UK) with a 64-channel analogue card was used to collect both the position of these markers and the force plate data at a sampling rate of $500 \mathrm{~Hz}$. Kinematic and force plate data were acquired and synchronised using the Vicon acquisition software (Workstation, Version 5.1 , Oxford, UK).

\subsection{Data Analysis}

After data collection, kinematic and force plate data were processed offline using a custom-made program written in MATLAB ${ }^{\text {TM }}$ (Version 5.3 (R11), The MathWorks Inc., Natick, MA, USA). Data were low-pass filtered using a Butterworth filter with a $15 \mathrm{~Hz}$ cutoff frequency for kinematic data [23] and a $10 \mathrm{~Hz}$ cutoff frequency for kinetic data [24]. The ML and anteroposterior (AP) coordinates of the centre of pressure ( $\mathrm{yP}$ and $\mathrm{xP}$, respectively) were computed from force plate data in accordance with the instructions from the manufacturer (AMTI) as follows:

$$
\begin{gathered}
\mathrm{yP}=\frac{\mathrm{Mx}+\mathrm{Fy} \times d z}{F z}, \\
\mathrm{xP}=\frac{-\mathrm{My}+\mathrm{Fx} \times d z}{\mathrm{Fz}},
\end{gathered}
$$

where Mx and My are the moment around the AP and ML axes, respectively; Fy, Fx and Fz are the ML, $\mathrm{AP}$ and vertical ground reaction forces, respectively; and $d \mathrm{z}$ is the distance between the surface of the force plate and its origin.

Instantaneous acceleration of the centre of mass along the AP, ML and vertical axes was determined from the ground reaction force according to Newton's second law. The centre of mass velocity and displacement were computed by the successive numerical integration of centre of mass acceleration using integration constants equal to zero, namely, initial velocity and displacement null [3].

Gait initiation onset $\left(\mathrm{t}_{0}\right)$ and swing-foot contact were determined from force plate data. Since gait initiation onset did not necessarily occur simultaneously in the ML and AP axes, two $\mathrm{t}_{0}$ times were estimated, one for each axis. $\mathrm{T}_{0 \mathrm{y}}$ and $\mathrm{t}_{0 \mathrm{x}}$ corresponded, respectively, to the instants when the ML and AP centre of pressure trace exceeded 2.5 standard deviations from its baseline value, respectively [24]. The one occurring first was considered $t_{0}$. Swing-foot contact corresponded to the instant when the ML and the AP centre of pressure trace shifted abruptly towards the swing-leg side and forward, respectively. Swing heel-off and swing toe-off were determined from VICON data. They corresponded to the instants when the swing heel marker's vertical position and the swing toe marker's anterior position exceeded their position in the initial static posture by $3 \mathrm{~mm}$.

\subsection{Dependant Variables}

Initial posture, APAs and swing foot lift. Gait initiation was divided into three phases, namely APAs (from $t_{0}$ to swing heel-off), swing foot lift (from heel-off to toe-off) and swing phase (from swing toe-off to swing foot contact) (Figure 2). The ML and AP centre of mass position in the initial upright posture was estimated by averaging the centre of pressure position during a $250 \mathrm{~ms}$ period, lasting from $\mathrm{t}_{0}$ minus $1500 \mathrm{~ms}$ to $\mathrm{t}_{0}$ minus $1250 \mathrm{~ms}$. No dynamic phenomena were detected during this period. APAs amplitude was characterised by the peaks of the backward and ML centre of pressure shift obtained during the APAs time window. The duration of APAs along the ML and AP axes was 
computed separately, as the $t_{0}$ times for these two axes did not necessarily occur simultaneously as stated above. The centre of mass velocity and displacement along the ML and AP axes were determined at swing toe-off and foot contact.

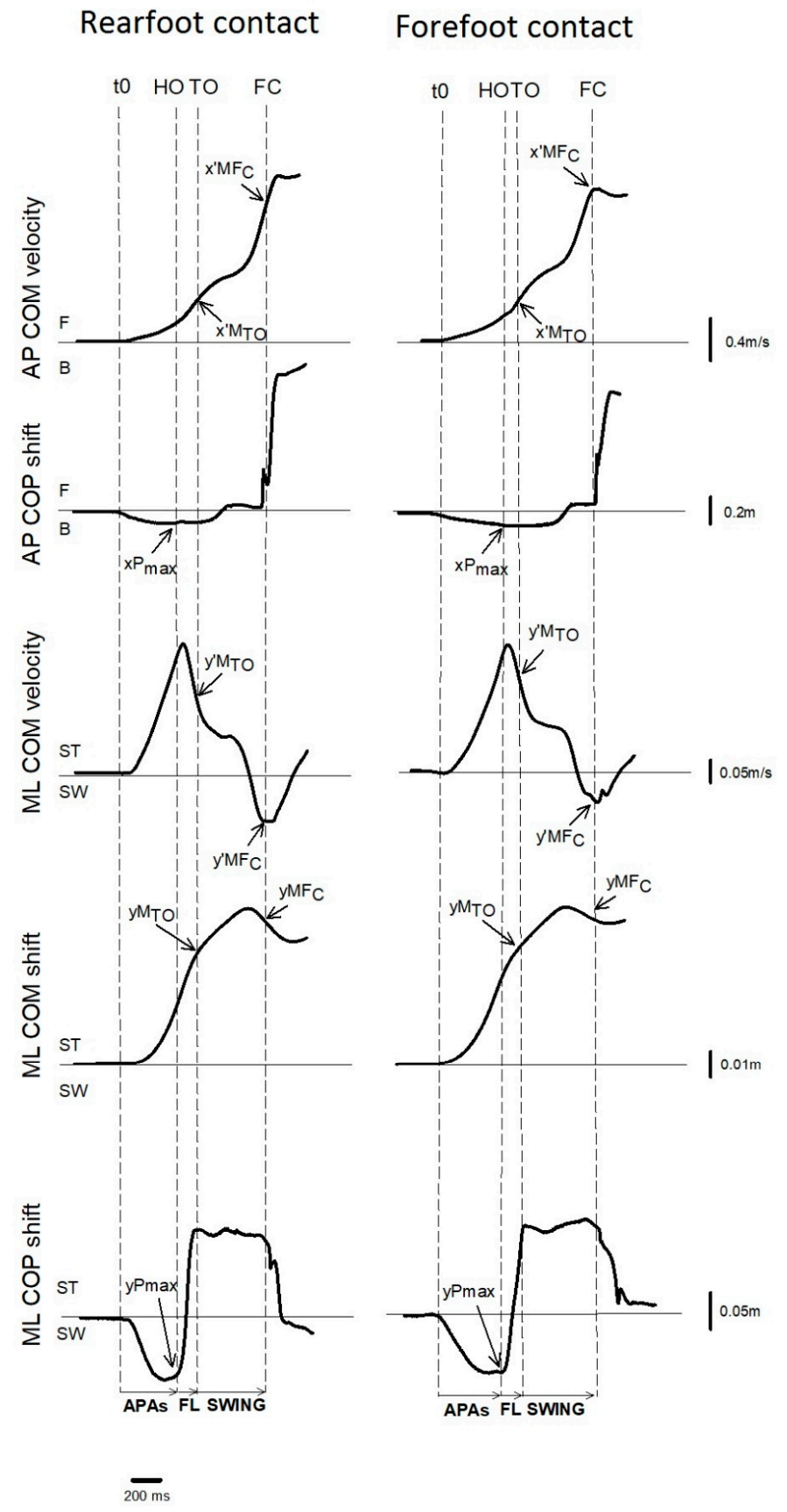

Figure 2. Typical biomechanical traces and representation of the main experimental variables (horizontal plane). Traces are from one representative subject initiating gait in the (left) rearfoot strike condition and the (right) forefoot strike (FFS) condition. Anteroposterior (AP) direction. $x^{\prime} \mathrm{M}_{\mathrm{TO}}, \mathrm{x}^{\prime} \mathrm{M}_{\mathrm{FC}}$ : centre of mass (COM) velocity at toe-off and foot contact; $x$ Pmax: peak of centre of pressure (COP) displacement during anticipatory postural adjustments (APAs); F: forward; B: backward. Mediolateral (ML) direction. $\mathrm{y}^{\prime} \mathrm{M}_{\mathrm{TO}}, y^{\prime} M_{F C}$ : COM velocity at toe-off and foot contact; $\mathrm{yM}_{\mathrm{TO}}, y M_{F C}$ : COM displacement at toe-off and foot contact; yPmax: peak of COP displacement during APAs; ST: stance limb; SW: swing limb. Vertical dashed lines: $t_{0}$ onset variation of biomechanical traces; HO: swing heel-off; TO: swing toe-off; FC: swing foot contact. Horizontal arrows. APAs: anticipatory postural adjustments; FL: swing foot lift; SWING: swing phase of gait initiation. 
Motor performance. Motor performance was quantified with the AP centre of mass velocity at foot contact, step length and swing phase duration. Step length corresponded to the distance covered by the heel marker of the swing leg from the initial posture to foot contact.

Safety distance. The vertical distance between the obstacle and the marker of the swing/rear heel, and between the obstacle and the marker of the swing/rear toe was measured at the instant these markers passed over the obstacle. The smaller of these two vertical distances corresponded to the safety distance.

ML dynamic stability. ML dynamic stability at foot contact (hereafter called ML stability) was quantified by the ML boundary of the base of support $\left(\mathrm{BOS}_{\mathrm{ymax}}\right)$ and an adaptation of the margin of stability (MOS) [25]. The BOS $\mathrm{y}_{\mathrm{y}}$ was measured as the distance at foot contact between the marker on the fifth metatarsal of the stance foot and the heel marker of the swing foot in the case of a rearfoot strike, or the marker on the fifth metatarsal of the swing foot in the case of a forefoot strike. The MOS corresponded to the difference between $\mathrm{BOS}_{\mathrm{ymax}}$ and the ML position of the extrapolated centre of mass at swing foot contact $\left(Y_{C O M} M_{F C}\right)$, that is, $M O S=B S_{y m a x}-Y_{c o} M_{F C}$. Based on the study of Hof et al. [25], the ML position of the extrapolated centre of mass at foot contact $\left(Y_{C o} M_{F C}\right)$ was calculated as follows:

$$
Y_{C O} M_{F C}=y M_{F C}+\frac{y^{\prime} M_{F C}}{\omega_{0}},
$$

where $y M_{F C}$ and $y^{\prime} M_{F C}$ are, respectively, the ML centre of mass position and velocity at foot contact, and $\omega_{0}$ is the eigenfrequency of the body modelled as an inverted pendulum calculated as $\omega_{0}=\sqrt{\frac{g}{l}}$, where $g=9.81 \mathrm{~m} / \mathrm{s}^{2}$ is gravitational acceleration and $l$ is the length of the inverted pendulum, which in this study was estimated to $57.5 \%$ of body height [26]. This quantity was held constant during the gait initiation. ML stability at foot contact is preserved on the condition that $Y{ }_{C O M} M_{F C}$ is within $B_{\text {OSmax }}$ which corresponds to a positive MOS.

Centre of mass braking. The braking index introduced by Welter and colleagues provides evidence that the centre of mass does not merely fall under the force of gravity but that the CNS prepares for foot contact by decreasing the centre of mass vertical velocity to achieve a soft landing [15]. It was calculated according to the formula of Chong et al. [12] as follows:

$$
\text { Braking index }=\left(\mathrm{V}_{\mathrm{MIN}}-\mathrm{V}_{\mathrm{FC}}\right) / \mathrm{V}_{\mathrm{MIN}}
$$

where $V_{\text {MIN }}$ is the minimum vertical centre of mass velocity occurring between mid- to late swing, and $\mathrm{V}_{\mathrm{FC}}$ is the vertical centre of mass velocity at foot contact (Figure 3 ).

This index indicates the amount of change (i.e., braking) in the centre of mass vertical velocity at foot contact relative to its minimal value. If the centre of mass vertical velocity is actively decreased before foot contact, the braking index value is increasing.

Collision forces. The combined effect of the foot strike pattern (RFS vs. FFS) and vertical force braking on the collision forces and on the transmission of these forces through the body was assessed using variables extracted from force plate and VICON data, respectively. The variables obtained from the force plate were the peak and slope of the vertical ground reaction force trace occurring immediately after foot contact [16] (Figure 3). The variable obtained from VICON data was the slope (computed at foot contact) of the vertical acceleration of the marker placed at the $\mathrm{C} 7$ spine level. 


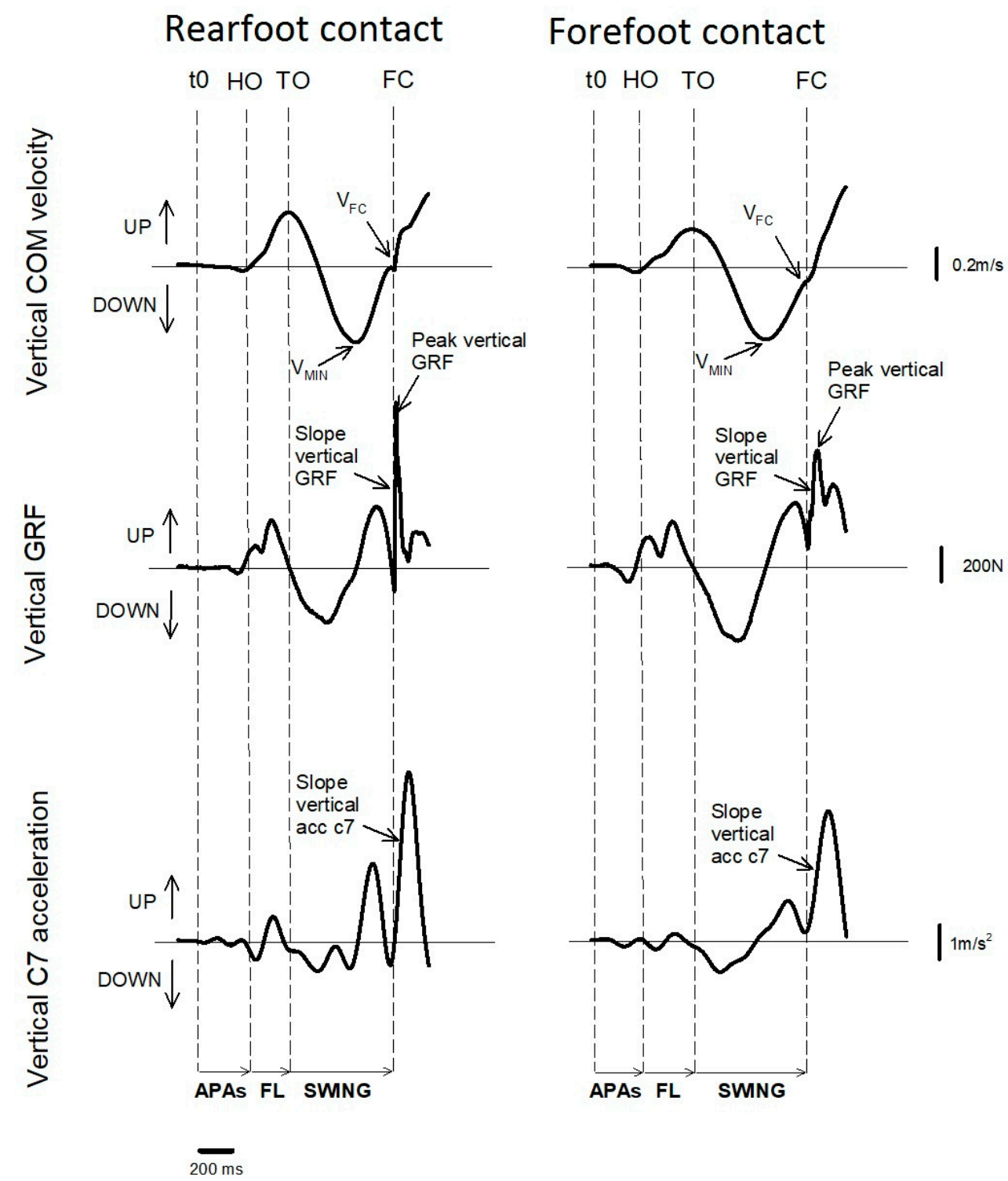

Figure 3. Typical biomechanical traces and representation of the main experimental variables (vertical direction). Traces are from one representative subject initiating gait in the (left) rearfoot strike condition and the (right) forefoot strike condition. COM, GRF, acc: centre of mass, ground reaction forces, and acceleration, respectively; $\mathrm{V}_{\mathrm{MIN}}, \mathrm{V}_{\mathrm{FC}}$ : peak of negative vertical COM velocity and COM velocity at foot contact (FC). Vertical dashed lines: $t_{0}$ onset variation of biomechanical traces; HO: swing heel-off; TO: swing toe-off; FC: swing foot contact. Horizontal arrows. APAs: anticipatory postural adjustments; FL: swing foot lift; SWING: swing phase of gait initiation.

\subsection{Statistics}

Mean values and standard errors were calculated for each variable under each condition. The normality of data was checked using the Kolmogorov-Smirnov test and the homogeneity of variances was assessed using Bartlett's test. Paired $t$-tests were used to compare mean values. The alpha level was set at 0.05 . 


\section{Results}

\subsection{Biomechanical Traces}

The time course of the biomechanical traces was generally similar under both experimental conditions (see Figures 2 and 3). Swing heel-off was systematically preceded by postural dynamics that corresponded to APAs. During these APAs, the centre of pressure displacement reached a peak value in a backward direction and towards the swing leg side, while the centre of mass velocity was directed forwards and towards the stance leg side. The ML centre of mass velocity trace reached a first peak value towards the stance leg side at around heel-off. This trace then fell towards the swing leg side and a second peak value towards this side was reached a few milliseconds after foot contact. The ML centre of mass shift trace was bell-shaped and reached a peak value towards the stance leg side in the late part of the swing phase. The AP centre of mass velocity increased progressively until it reached a peak value a few milliseconds after swing foot contact, while the centre of mass was continuously shifted forward (see Figure 2).

The time course of the vertical ground reaction force and the centre of mass vertical velocity was also generally similar under both experimental conditions (see Figure 3). Following foot-off, the centre of mass was accelerated downwards (negative velocity indicates downward centre of mass movement) and then reversed. In fact, during single support, the centre of mass velocity shows a $\mathrm{V}$ shape indicating that the centre of mass fall was braked.

\subsection{Initial Posture, APAs and Swing Foot Lift}

Statistical analysis showed that there was no significant effect of the swing-foot strike pattern on the initial position of the centre of mass along the ML and the AP direction. Additionally, there was no significant effect of the swing-foot strike pattern on the peak of AP centre of pressure shift and AP/ML APAs duration (Figure 4). By contrast, the peak of the ML centre of pressure shift was significantly smaller under the FFS than under the RFS condition $\left(t_{12}=-2.43, p=0.032\right)$ and foot lift duration was significantly longer under the FFS than under the RFS condition $\left(\mathrm{t}_{12}=5.22, p<0.001\right)$. Foot lift duration was $88 \pm 8 \mathrm{~ms}$ under the RFS condition and $105 \pm 7$ ms under the FFS condition. Finally, the ML centre of mass velocity at toe-off was significantly lower under the FFS than under the RFS condition $\left(\mathrm{t}_{12}=-3.25, p=0.006\right)$, whereas the AP centre of mass velocity at toe-off was significantly higher $\left(\mathrm{t}_{12}=2.46, p=0.003\right)$.

\subsection{Safety Distance}

The safety distance values for the swing foot and for the rear foot were $13.6 \pm 0.9 \mathrm{~cm}$ and $14.4 \pm$ $1.4 \mathrm{~cm}$, respectively, under the RFS condition, and were $13.7 \pm 0.9 \mathrm{~cm}$ and $15.0 \pm 1.5 \mathrm{~cm}$, respectively, under the FFS condition, with no significant difference across conditions.

\subsection{Motor Performance}

The swing phase duration was significantly shorter $\left(\mathrm{t}_{12}=-5.01, p<0.001\right)$ and the AP centre of mass velocity at foot contact was significantly smaller $\left(\mathrm{t}_{12}=-4.64, p<0.001\right)$ under the FFS than under the RFS condition. By contrast, step length did not differ between the two conditions. Step length was $79.2 \pm 4.0 \mathrm{~cm}$ under the RFS condition and $77.8 \pm 4.5 \mathrm{~cm}$ under the FFS condition.

\subsection{Dynamic Stability}

The ML margin of stability (MOS) at foot contact was significantly greater under the FFS than under the RFS condition $\left(\mathrm{t}_{12}=6.12, p<0.001\right)$, showing that ML dynamic stability at foot contact was increased under the FFS compared to the RFS condition (Figure 5). The results further show that the ML size of the base of support (BOS ${ }_{\text {ymax }}$ ) was larger under the FFS than under the RFS condition $\left(\mathrm{t}_{12}=4.77, p<0.001\right)$, and that the ML centre of mass velocity at foot contact was lower $\left(\mathrm{t}_{12}=5.75\right.$, 
$p<0.001)$. By contrast, the ML centre of mass position at foot contact was not significantly different between the two conditions.
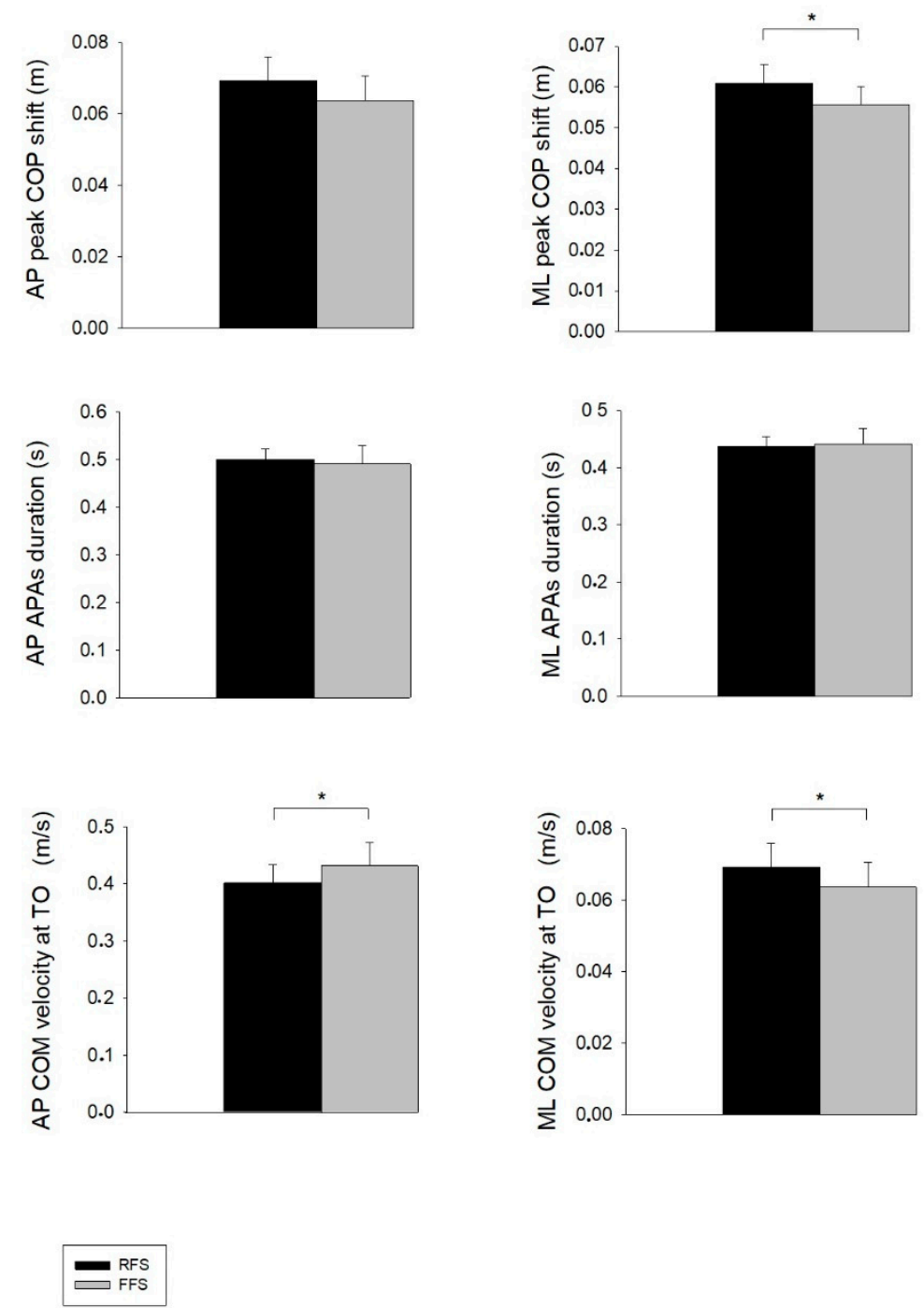

Figure 4. Effects of swing-foot strike pattern on selected APA parameters. Reported are mean values (all participants together) + 1 SE. APAs: anticipatory postural adjustments; TO: toe-off; COP: centre of pressure; COM: centre of mass; RFS: rearfoot strike condition; FFS: forefoot strike condition. Bars at the left side: mediolateral (ML) direction. Bars at the right side: anteroposterior (AP) direction. * Indicates a significant difference between bars.

\subsection{Centre of Mass Braking}

The braking index was significantly smaller under the FFS than under the RFS condition $\left(\mathrm{t}_{12}=-2.44\right.$, $p=0.03$ ), that is, the downward vertical centre of mass velocity was braked less before foot contact under the FFS condition (Figure 6). As a consequence, the vertical centre of mass velocity at foot contact was greater under the FFS than under the RFS condition $\left(t_{12}=-2.19, p=0.049\right)$, and the swing phase was shorter (as reported above). 

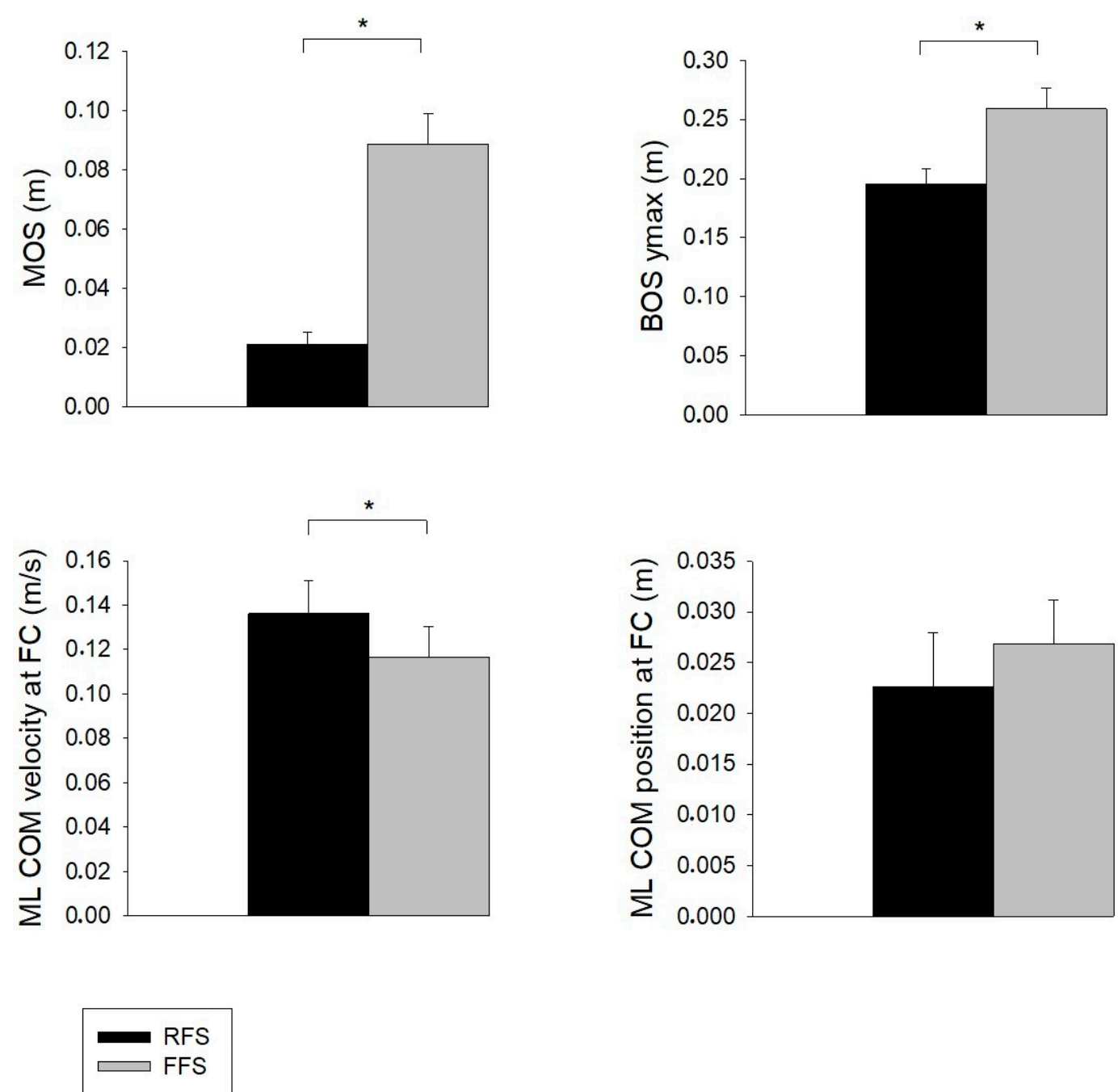

Figure 5. Effects of swing-foot strike pattern on selected stability parameters along the mediolateral (ML) direction. Reported are mean values (all participants together) + 1 SE. BOSymax: base of support size; MOS: margin of stability; COM: centre of mass; RFS: rearfoot strike condition; FC: swing foot contact; FFS: forefoot strike condition. * Indicates a significant difference between bars.

\subsection{Collision Forces}

The peak value of the vertical ground reaction forces, which occurred shortly after foot contact and reflected the collision forces acting at the foot, was significantly smaller under the FFS than under the RFS condition $\left(\mathrm{t}_{12}=-5.61, p<0.001\right)$, as was the slope of these forces $\left(\mathrm{t}_{12}=-5.11, p<0.001\right.$; see Figure 6). In addition, the slope of the vertical acceleration (computed at foot contact) of the marker located at the C7 spine level was significantly smaller under the FFS than under the RFS condition $\left(\mathrm{t}_{12}=-2.32, p=0.045\right)$. 

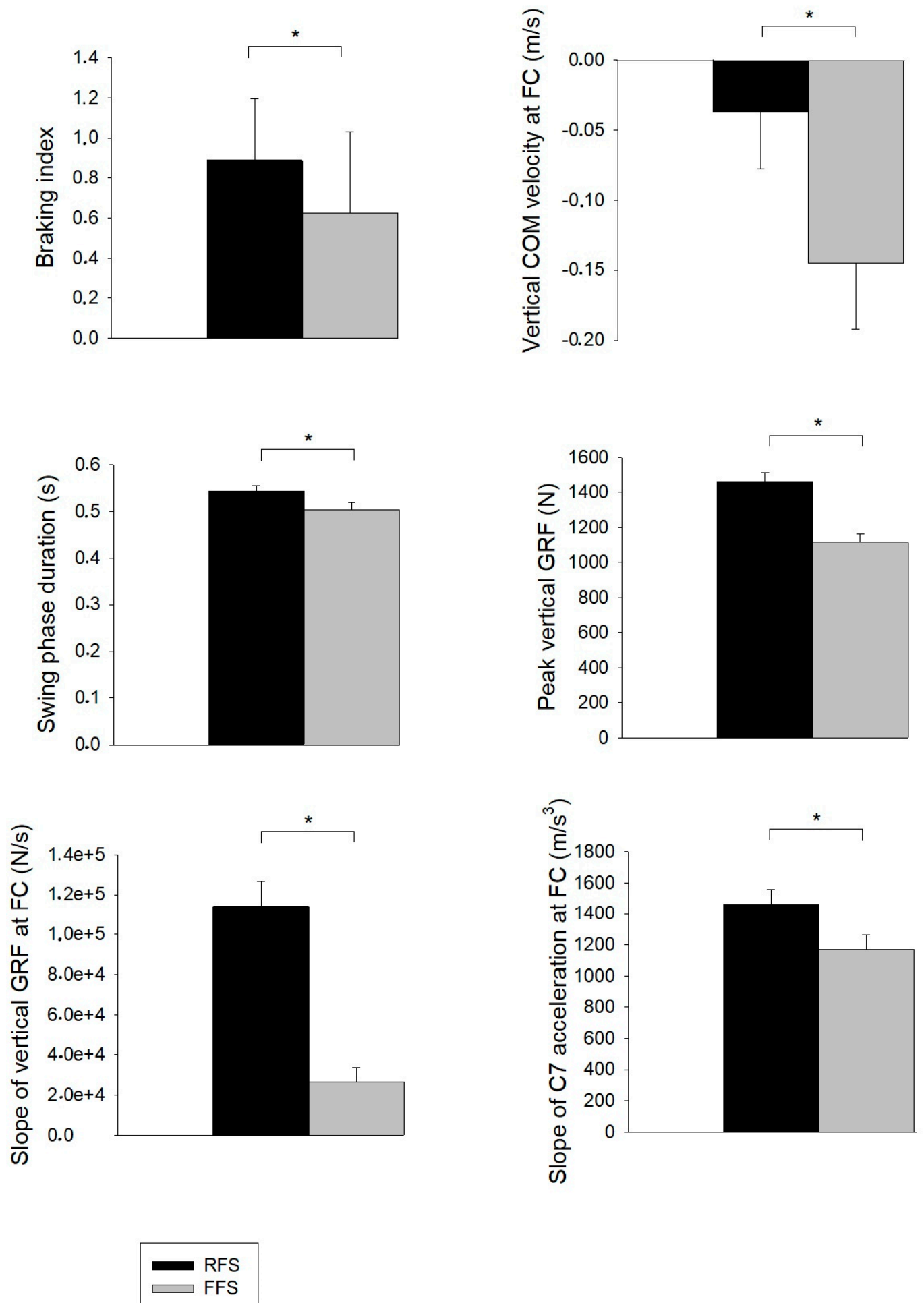

Figure 6. Effects of swing-foot strike pattern on collision force parameters, vertical force braking and swing duration. Reported are mean values (all participants together) \pm 1 SE. GRF: ground reaction forces; COM: centre of mass; C7: 7th cervical vertebrae; FC: foot contact; RFS: rearfoot strike condition; FFS: forefoot strike condition. * Indicates a significant difference between bars.

\section{Discussion}

The present study investigated the effect of changing the swing-foot strike pattern (FFS vs. RFS) on ML APAs and active braking of the centre of mass fall, and on the related ML stability and force 
collisions. Specifically, it was hypothesised that the active braking of the centre of mass fall and the ML APAs associated with gait initiation over an obstacle to be cleared are both attenuated in an FFS compared to an RFS pattern, but with no alteration of ML stability or increase in collision forces. The results discussed below are in line with these expectations and suggest the existence of an interdependent relationship between balance control mechanisms for optimal stability control.

Do and colleagues first reported that, in young healthy adults, the centre of mass fall during the swing phase of gait initiation was actively braked in anticipation of the swing foot's collision with the ground $[2,14,15,27,28]$. This centre of mass brake, which was ascribed to an activation of the soleus of the stance leg (not recorded in the present study), aims to attenuate the vertical ground reaction forces at the time of foot contact (i.e., the collision forces). Therefore, this braking facilitates balance control following foot contact and attenuates the transmission of the collision forces throughout the entire musculoskeletal system by allowing a softer swing foot landing $[6,14,15]$. The results of the present study showed that the indicator of this centre of mass brake (the braking index) reached a significantly lower value under the FFS than under the RFS condition (the difference between the two conditions was $\Delta=19.4 \%$ ), thus revealing that the fall of the centre of mass was much less braked under the FFS condition. The much larger downward-oriented centre of mass velocity at foot contact under the FFS condition $(\Delta=74.5 \%)$ can be considered a direct consequence of this reduced braking. Therefore, it could be surprising that the two indicators of the collision forces' perturbing effect (namely, peak collision forces and the slope of this peak) were both smaller under the FFS than under the RFS condition $(\Delta=27.3 \%$ and $\Delta=26.2 \%$, respectively), revealing that the foot landing was softer under the first condition. In addition, the jerk value recorded at the C7 spine level was lower under the FFS than under the RFS condition. This finding suggests that the collision forces acting at the foot were absorbed to a greater extent by the swing leg under the FFS condition, reducing its transmission through the upper part of the musculoskeletal system. In turn, these results are in line with classical studies in the literature comparing collision forces during running [16,19-22,29,30], or during stepping down during ongoing gait [17]. According to van Dieën et al. [17], the FFS pattern allows the ankle plantar flexors of the swing leg to produce negative work that can absorb part of the collision forces. This negative work is absent in the RFS pattern which might explain the greater collision forces found under the RFS condition. As stressed in the introduction, it is noteworthy that centre of mass braking has not been investigated in any of these studies. Consequently, a possible modulation effect of this force braking on the collision forces cannot be discounted in these studies.

In fact, the result of the present study showing that the collision forces were smaller under the FFS than under the RFS condition, despite the centre of mass braking being attenuated, further stresses the efficiency of the FFS pattern in dampening collision forces. The present results may also suggest that the dampening effect specifically associated with the FFS alone was probably underestimated in the studies on foot strike pattern cited above.

The question arises as to why centre of mass braking is attenuated under the FFS condition compared to the RFS condition. To interpret this finding, it should be stressed that this braking has an energetic and attentional cost $[14,15]$ and shares a dampening action on collision forces with the foot strike strategy. Therefore, when the FFS pattern is employed, the need to actively brake the centre of mass fall becomes less crucial than when the RFS pattern is used. The CNS would then attenuate this active brake in order to minimise the associated energetic and attentional cost, and would rely more heavily on the efficiency of the foot strike strategy to dampen the collision forces. This implies that the CNS takes into account the foot strike pattern and the related dampening effect when programming braking force generation. This statement is in line with the hypothesis of an interdependent relationship between these two mechanisms (active braking and foot strike pattern) for optimal stability control and better ground reaction force management. The results linking ML APAs to the foot strike pattern further strengthen this hypothesis (paragraph below).

During gait initiation or any locomotor task, imbalance occurs in the frontal plane due to the transition from a bipedal to a unipedal stance. Following swing foot-off, the centre of mass falls 
rapidly towards the swing leg side under gravity, and this lateral fall is braked by foot contact with the ground. This ML imbalance is greater when an obstacle is to be cleared, since the duration of the lateral fall of the centre of mass is increased compared to a control condition with no obstacle to be cleared [10,11,31-33]. The development of ML APAs is therefore of utmost importance to maintain stability as it acts to minimise this lateral fall by propelling the centre of mass towards the stance leg side and thus attenuating the ML gap between the centre of mass and the centre of pressure at the time of swing foot-off $[7,8,10,11,34,35]$. Previous modelling studies demonstrated the link between ML APAs and ML stability at foot contact $[8,10,11,34]$.

In the present study, the results showed that the amplitude of these ML APAs, in terms of peak centre of pressure shift, was lower under the FFS than under the RFS condition $(\Delta=9.4 \%)$. It also revealed that this lower amplitude was not accompanied by a ML APA duration lengthening strategy. Such a trade-off strategy between APA amplitude and APA duration has been repeatedly reported in the literature under various conditions of postural constraints, such as fear of falling [36], fatigue [37-39], leg dominance [40] and temporal pressure [41]. There was no such compensative trade-off strategy in the present study. Consequently, the ML centre of mass velocity at the time of swing foot-off, which represents the total amount of forces applied to the centre of mass during the APAs, reached a lower value under the FFS than under the RFS condition $(\Delta=5.99 \%)$. For the above reasons, ML dynamic stability, as measured with the MOS [25], could be expected to be lower under the FFS than under the RFS condition. However, contrary to this expectation, the MOS reached much higher values under the FFS than under the RFS condition $(\Delta=71.42 \%)$, that is, ML dynamic stability was significantly improved. Changes in two components of the MOS with the foot strike strategy can account for this result, as detailed below.

First, the ML centre of mass velocity at the time of foot contact reached a lower value under the FFS than under the RFS condition $(\Delta=14 \%)$, that is, the velocity at which the centre of mass fell towards the swing leg side was attenuated. This finding could be ascribed to the lower duration of the swing phase of gait initiation under the FFS compared to the RFS condition $(\Delta=6.70 \%)$, which implies that the period during which the centre of mass was falling laterally under gravity was reduced. In turn, this shorter duration of the swing phase under the FFS condition could be ascribed to the lower force braking under the FFS condition $(\Delta=19.4 \%)$, which accelerated the fall of the centre of mass to a greater extent (as discussed above).

As a second factor contributing to enhanced stability, the size of the ML base of support was increased under the FFS compared to the RFS condition. This result could not be ascribed to a strategy of a more lateral placement of the swing foot $[24,35]$ (since the distance between the two heels remained unchanged between the two foot strike conditions), but to foot anatomy. Under the FFS condition, the ML base of support size corresponded to the distance between the stance heel and the fifth metatarsal of the swing foot, while under the RFS condition, it corresponded to the distance between the two heels. It was therefore smaller than under the FFS condition. This difference reached up to $10 \mathrm{~cm}$ in some subjects.

The question arises as to why the amplitude of ML APAs is decreased when stability is increased with the change in the foot strike pattern.

Generating ML APAs is known to have an energetic cost, requiring a coordinated activation of hip abductors and ankle dorsiflexors [7,42]. Although not shown in the literature to date, it may also have an attentional cost, as has previously been documented for the control of static erect posture and level walking $[43,44]$. An optimal strategy for stability control would appear to be to reduce this cost by attenuating the ML APAs under the FFS condition while, at the same time, increasing stability with the mechanical and anatomical changes reported above-neither of which requires any additional energetic or attentional cost (even less for attenuated force braking). This optimal strategy implies that one (or both) of these factors associated with the foot strike pattern be accounted for in the programming of ML APAs. This statement further reinforces the hypothesis proposed above of an interdependent relationship between motor mechanisms for optimal stability control. 
Interestingly, previous studies focusing on the effect of different sorts of constraints applied to the postural system during gait initiation (such as temporal pressure [41,45], progression velocity [24], the presence of an obstacle of different heights and distances to be cleared $[10,11]$, the addition of a mass distributed (a)symmetrically over the body or the ankles [46-48], etc.) repeatedly showed that the CNS modulated the spatiotemporal features of ML APAs so as to maintain an equivalent MOS value. This motor invariance through these disparate postural constraints led the authors to suggest that the CNS would set a fixed MOS value before initiating gait and would then adjust ML APAs features to keep it unchanged (for review, see [2]). Thus, the present finding that the MOS was not invariant is not in line with these previous results and related hypothesis. Now, it may be recalled that the participants systematically used an RFS pattern in all of the previous studies. Therefore, the MOS was computed and compared across conditions at the moment the swing heel hit the ground. By contrast, the present study compared the MOS of two different postures of the landing foot. These foot postures were responsible for the difference in the ML size of the base of support, which was a major factor explaining the MOS differences between the two foot strike conditions. It is possible that, for a given foot strike pattern (RFS or FFS), a fixed MOS value is planned before initiating gait, and may be greater for the FFS than the RFS pattern due to foot anatomy.

\section{Conclusions}

In conclusion, these findings are in line with the hypothesis of an interdependent relationship between balance control mechanisms and foot strike pattern for optimal stability control. These findings are original since previous studies in the literature focused on either balance control mechanisms during gait initiation or changes in the collision forces with the foot strike pattern. Thus, the present study linked these two major lines of research to better highlight how balance is controlled in humans during a functional locomotor task. The question remains as to whether and how coordination between these balance control mechanisms is affected by aging as well as neurological and musculoskeletal disorders.

Author Contributions: R.A., P.F., C.T., T.C., A.D., E.Y.: made substantial contributions to the conception of the work, the acquisition, analysis, and interpretation of data; Drafted the work and revised it critically; made final approval of the version to be published; Agreed to be accountable for all aspects of the work in ensuring that questions related to the accuracy or integrity of any part of the work are appropriately investigated and resolved. All authors have read and agreed to the published version of the manuscript.

Funding: This research received no external funding.

Conflicts of Interest: The authors declare no conflict of interest.

\section{References}

1. Yiou, E.; Caderby, T.; Hussein, T. Adaptability of anticipatory postural adjustments associated with voluntary movement. World J. Orthop. 2012, 3, 75-86. [CrossRef] [PubMed]

2. Yiou, E.; Caderby, T.; Delafontaine, A.; Fourcade, P.; Honeine, J.-L. Balance control during gait initiation: State-of-the-art and research perspectives. World J. Orthop. 2017, 8, 815-828. [CrossRef] [PubMed]

3. Brenière, Y.; Cuong Do, M.; Bouisset, S. Are dynamic phenomena prior to stepping essential to walking? J. Mot. Behav. 1987, 19, 62-76. [CrossRef] [PubMed]

4. Breniere, Y.; Do, M.C. When and how does steady state gait movement induced from upright posture begin? J. Biomech. 1986, 19, 1035-1040. [CrossRef]

5. Lepers, R.; Brenière, Y. The role of anticipatory postural adjustments and gravity in gait initiation. Exp. Brain Res. 1995, 107, 118-124. [CrossRef]

6. Michel, V.; Chong, R.K.Y. The strategies to regulate and to modulate the propulsive forces during gait initiation in lower limb amputees. Exp. Brain Res. 2004, 158, 356-365. [CrossRef]

7. Honeine, J.-L.; Schieppati, M.; Crisafulli, O.; Do, M.-C. The Neuro-Mechanical Processes That Underlie Goal-Directed Medio-Lateral APA during Gait Initiation. Front. Hum. Neurosci. 2016, 10, 445. [CrossRef]

8. Lyon, I.N.; Day, B.L. Control of frontal plane body motion in human stepping. Exp. Brain Res. 1997, 115, 345-356. [CrossRef] 
9. McIlroy, W.E.; Maki, B.E. The control of lateral stability during rapid stepping reactions evoked by antero-posterior perturbation: Does anticipatory control play a role? Gait Posture 1999, 9, 190-198. [CrossRef]

10. Yiou, E.; Fourcade, P.; Artico, R.; Caderby, T. Influence of temporal pressure constraint on the biomechanical organization of gait initiation made with or without an obstacle to clear. Exp. Brain Res. 2016, 234, 1363-1375. [CrossRef]

11. Yiou, E.; Artico, R.; Teyssedre, C.A.; Labaune, O.; Fourcade, P. Anticipatory Postural Control of Stability during Gait Initiation Over Obstacles of Different Height and Distance Made Under Reaction-Time and Self-Initiated Instructions. Front. Hum. Neurosci. 2016, 10, 449. [CrossRef] [PubMed]

12. Chong, R.K.Y.; Chastan, N.; Welter, M.-L.; Do, M.-C. Age-related changes in the center of mass velocity control during walking. Neurosci. Lett. 2009, 458, 23-27. [CrossRef] [PubMed]

13. Delafontaine, A.; Gagey, O.; Colnaghi, S.; Do, M.-C.; Honeine, J.-L. Rigid Ankle Foot Orthosis Deteriorates Mediolateral Balance Control and Vertical Braking during Gait Initiation. Front. Hum. Neurosci. 2017, 11, 214. [CrossRef] [PubMed]

14. Honeine, J.-L.; Schieppati, M.; Gagey, O.; Do, M.-C. By counteracting gravity, triceps surae sets both kinematics and kinetics of gait. Physiol. Rep. 2014, 2, e00229. [CrossRef] [PubMed]

15. Welter, M.-L.; Do, M.C.; Chastan, N.; Torny, F.; Bloch, F.; du Montcel, S.T.; Agid, Y. Control of vertical components of gait during initiation of walking in normal adults and patients with progressive supranuclear palsy. Gait Posture 2007, 26, 393-399. [CrossRef]

16. Lieberman, D.E.; Venkadesan, M.; Werbel, W.A.; Daoud, A.I.; D’Andrea, S.; Davis, I.S.; Mang'eni, R.O.; Pitsiladis, Y. Foot strike patterns and collision forces in habitually barefoot versus shod runners. Nature 2010, 463, 531-535. [CrossRef]

17. van Dieën, J.H.; Spanjaard, M.; Könemann, R.; Bron, L.; Pijnappels, M. Mechanics of toe and heel landing in stepping down in ongoing gait. J. Biomech. 2008, 41, 2417-2421. [CrossRef]

18. van Dieën, J.H.; Pijnappels, M. Effects of conflicting constraints and age on strategy choice in stepping down during gait. Gait Posture 2009, 29, 343-345. [CrossRef]

19. Almeida, M.O.; Davis, I.S.; Lopes, A.D. Biomechanical Differences of Foot-Strike Patterns During Running: A Systematic Review With Meta-analysis. J. Orthop. Sports Phys. Ther. 2015, 45, 738-755. [CrossRef]

20. Boyer, E.R.; Rooney, B.D.; Derrick, T.R. Rearfoot and midfoot or forefoot impacts in habitually shod runners. Med. Sci. Sports Exerc. 2014, 46, 1384-1391. [CrossRef]

21. Breine, B.; Malcolm, P.; Van Caekenberghe, I.; Fiers, P.; Frederick, E.C.; De Clercq, D. Initial foot contact and related kinematics affect impact loading rate in running. J. Sports Sci. 2017, 35, 1556-1564. [CrossRef] [PubMed]

22. Hatala, K.G.; Dingwall, H.L.; Wunderlich, R.E.; Richmond, B.G. Variation in foot strike patterns during running among habitually barefoot populations. PLoS ONE 2013, 8, e52548. [CrossRef] [PubMed]

23. Mickelborough, J.; van der Linden, M.L.; Richards, J.; Ennos, A.R. Validity and reliability of a kinematic protocol for determining foot contact events. Gait Posture 2000, 11, 32-37. [CrossRef]

24. Caderby, T.; Yiou, E.; Peyrot, N.; Begon, M.; Dalleau, G. Influence of gait speed on the control of mediolateral dynamic stability during gait initiation. J. Biomech. 2014, 47, 417-423. [CrossRef]

25. Hof, A.L.; Gazendam, M.G.J.; Sinke, W.E. The condition for dynamic stability. J. Biomech. 2005, 38, 1-8. [CrossRef]

26. Winter, D. Human balance and posture control during standing and walking. Gait Posture 1995, 3, $193-214$. [CrossRef]

27. Chastan, N.; Westby, G.W.M.; du Montcel, S.T.; Do, M.C.; Chong, R.K.; Agid, Y.; Welter, M.L. Influence of sensory inputs and motor demands on the control of the centre of mass velocity during gait initiation in humans. Neurosci. Lett. 2010, 469, 400-404. [CrossRef]

28. Honeine, J.-L.; Schieppati, M.; Gagey, O.; Do, M.-C. The Functional Role of the Triceps Surae Muscle during Human Locomotion. PLoS ONE 2013, 8, e52943. [CrossRef]

29. Cunningham, C.B.; Schilling, N.; Anders, C.; Carrier, D.R. The influence of foot posture on the cost of transport in humans. J. Exp. Biol. 2010, 213, 790-797. [CrossRef]

30. Daoud, A.I.; Geissler, G.J.; Wang, F.; Saretsky, J.; Daoud, Y.A.; Lieberman, D.E. Foot strike and injury rates in endurance runners: a retrospective study. Med. Sci. Sports Exerc. 2012, 44, 1325-1334. [CrossRef]

31. Chou, L.-S.; Kaufman, K.R.; Hahn, M.E.; Brey, R.H. Medio-lateral motion of the center of mass during obstacle crossing distinguishes elderly individuals with imbalance. Gait Posture 2003, 18, 125-133. [CrossRef] 
32. Chou, L.S.; Kaufman, K.R.; Brey, R.H.; Draganich, L.F. Motion of the whole body's center of mass when stepping over obstacles of different heights. Gait Posture 2001, 13, 17-26. [CrossRef]

33. Hahn, M.E.; Chou, L.-S. Age-related reduction in sagittal plane center of mass motion during obstacle crossing. J. Biomech. 2004, 37, 837-844. [CrossRef] [PubMed]

34. Lyon, I.N.; Day, B.L. Predictive control of body mass trajectory in a two-step sequence. Exp. Brain Res. 2005, 161, 193-200. [CrossRef] [PubMed]

35. Zettel, J.L.; McIlroy, W.E.; Maki, B.E. Environmental constraints on foot trajectory reveal the capacity for modulation of anticipatory postural adjustments during rapid triggered stepping reactions. Exp. Brain Res. 2002, 146, 38-47. [CrossRef] [PubMed]

36. Yiou, E.; Deroche, T.; Do, M.C.; Woodman, T. Influence of fear of falling on anticipatory postural control of medio-lateral stability during rapid leg flexion. Eur. J. Appl. Physiol. 2011, 111, 611-620. [CrossRef]

37. Mezaour, M.; Yiou, E.; Le Bozec, S. Effect of lower limb muscle fatigue on anticipatory postural adjustments associated with bilateral-forward reach in the unipedal dominant and non-dominant stance. Eur. J. Appl. Physiol. 2010, 110, 1187-1197. [CrossRef]

38. Vuillerme, N.; Nougier, V.; Teasdale, N. Effects of lower limbs muscular fatigue on anticipatory postural adjustments during arm motions in humans. J. Sports Med. Phys. Fit. 2002, 42, 289-294.

39. Yiou, E.; Ditcharles, S.; Le Bozec, S. Biomechanical reorganisation of stepping initiation during acute dorsiflexor fatigue. J. Electromyogr. Kinesiol. 2011, 21, 727-733. [CrossRef]

40. Mezaour, M.; Yiou, E.; Le Bozec, S. Does symmetrical upper limb task involve symmetrical postural adjustments? Gait Posture 2009, 30, 239-244. [CrossRef]

41. Yiou, E.; Hussein, T.; Larue, J. Influence of temporal pressure on anticipatory postural control of medio-lateral stability during rapid leg flexion. Gait Posture 2012, 35, 494-499. [CrossRef] [PubMed]

42. Nouillot, P.; Bouisset, S.; Do, M.C. Do fast voluntary movements necessitate anticipatory postural adjustments even if equilibrium is unstable? Neurosci. Lett. 1992, 147,1-4. [CrossRef]

43. Fraizer, E.V.; Mitra, S. Methodological and interpretive issues in posture-cognition dual-tasking in upright stance. Gait Posture 2008, 27, 271-279. [CrossRef] [PubMed]

44. Woollacott, M.; Shumway-Cook, A. Attention and the control of posture and gait: A review of an emerging area of research. Gait Posture 2002, 16,1-14. [CrossRef]

45. Hussein, T.; Yiou, E.; Larue, J. Age-related differences in motor coordination during simultaneous leg flexion and finger extension: Influence of temporal pressure. PLoS ONE 2013, 8, e83064. [CrossRef]

46. Caderby, T.; Yiou, E.; Peyrot, N.; de Viviés, X.; Bonazzi, B.; Dalleau, G. Effects of Changing Body Weight Distribution on ML Stability Control during Gait Initiation. Front. Hum. Neurosci. 2017, 11, 127. [CrossRef]

47. Caderby, T.; Dalleau, G.; Leroyer, P.; Bonazzi, B.; Chane-Teng, D.; Do, M.C. Does an additional load modify the Anticipatory Postural Adjustments in gait initiation? Gait Posture 2013, 37, 144-146. [CrossRef]

48. Yiou, E.; Hussein, T.; LaRue, J. Influence of ankle loading on the relationship between temporal pressure and motor coordination during a whole-body paired task. Exp. Brain Res. 2014, 232, 3089-3099. [CrossRef]

(C) 2019 by the authors. Licensee MDPI, Basel, Switzerland. This article is an open access article distributed under the terms and conditions of the Creative Commons Attribution (CC BY) license (http://creativecommons.org/licenses/by/4.0/). 